\title{
A Review of Techniques used in EPS and 4G-LTE in Mobility Schemes
}

\author{
Deepak N. R \\ Research Scholar, VTU \\ Dept of Computer Science \& Engg. \\ City Engineering College \\ Bangalore-560061, India
}

\author{
S. Balaji, Ph.D \\ Centre for Emerging Technologies \\ Jain University, Jain Global Campus \\ Jakkasandra Post, Kanakapura Taluk \\ Ramanagara Dist., Karnataka-562112, India
}

\begin{abstract}
With the extensive proliferation of mobile applications and smartphones, there is an increasing trend of technical adoption of $3 \mathrm{G}$ (third generation). But, with the exponential growing of massive traffic, it is seen that even $3 \mathrm{G}$ network is completely not capable of catering to the dynamic needs of the customers. This paper focuses on the usage of 4G-LTE (fourth generation-long term evolution) network that has higher support for heterogeneous network access catering to the needs. 4G-LTE is designed on the principles of packet switching system on the backbone of IP protocol. This paper presents a review on the state-of-art techniques from the viewpoint of mobility management approach, which is highly enriched with informative contents compared to existing reviews.
\end{abstract}

\section{Keywords}

3GPP, Evolving Packet System, Evolved-UTRAN (E-UTRAN), Long-Term Evolution, Mobile Networks, Universal Terrestrial Network (UTRAN)

\section{INTRODUCTION}

With the rise of adoption of smartphones, the area of telecommunication especially the mobile device and standards industry are encountering a new paradigm of technological advancement [1].The present paper has discussed the emergence of LTE (Long-Term Evolution) that acts as the standard for the wireless communication system for high-speed data transmission for catering to the needs of dynamic mobile users [2]. The concept of LTE is basically based on the existing technologies e.g. GSM/EDGE (Global System for Mobile Communications/ Enhanced Data Rates for GSM Evolution) as well as UMTS (Universal Mobile Telecommunications System) for the purpose of enhancing the capabilities of mobile network [3]. Different countries use different frequencies as well as bands for LTE network due to which a mobile device with support for multiband is required. Essentially, the architecture designed for LTE protocol consists of user plane and control plane [4]. The user plane is responsible for furnishing function between user device and Evolved Universal Terrestrial Radio Access (EUTRAN),(UMTS-Universal Mobile Telecommunication System) Terrestrial Radio Access Network) while control plane is used for providing the access policies [5]. The LTE network is essentially designed using Evolving Packet System (EPS) that consists of multiple radio access resources called and network of IP cores. The EPS is responsible for providing some of the essential services like voice-based communication, multimedia streaming and transmission adopting completely shared radio resource allocation scheme for enhancing the utility of radio resources. However, the upcoming trends of 4G-LTE have enhanced experience compared to $3 \mathrm{G}$ networks. Potential applications like accessing gaming services, IP telephony, video calls are highly supported in such networks [6]. Apart from this, it also supports the ubiquitous computing like cloud networks to get integrated along with it for giving better user experiences. Usually a $4 \mathrm{G}$ network is built from multiple wireless networks for supporting hand-off from one to other technologies. Wireless networks have their own advantages and limitations when such heterogeneous network is considered. Eventually LTE network is also associated with various issues [7]: e.g. i) network discovery, ii) access technologies, iii) network condition, iv) network architecture, v) charging and billing process, vi) security, vii) large number of operators, and viii) congestion control. In addition to such issues, there exist many more problems associated with the mobility management of $4 \mathrm{G}-$ LTE: e.g. i) inefficient QoS, ii) signaling overhead, iii) energy requirements, iv) less scalable and reliability in hand-off process.

Interestingly, the study towards mobile networks is a decade old. Majority of the studies focus on individual wireless network, where the actual focus on problem is make. As the present users are more inclined towards adopting smart mobile communication technologies, e.g. 4G-LTE, it is essential that a researcher must have clarity of the effectiveness in the existing techniques based on which the researcher can perform further investigation. Modeling wireless communication system with the standards of 3GPP-LTE considering heterogeneity of the access network is one of the challenging problems for investigating the effectiveness of the techniques. Even for the purpose of benchmarking, it is important that we must know the best framework proposed till date in LTE network that has better mitigation techniques of critical problems. This paper discusses about the prior research attempts by investigation and reviewing the effectiveness of the techniques for improving the performance of LTE networks. The prime objective of this paper is to excavate the research gap and emerging trends of LTE network in this area. Section 2 discusses about the important factors associated with 3GPP-LTE (third Generation Partnership Project) network followed by Section 3 that discusses about contribution of some of the recent studies. Discussion of emerging trends of research community is highlighted in Section 4. Section 5 discusses about research gap and finally in section 6 , we make some concluding remarks

\section{3GPP-LTE}

The concept of Evolved Packet System (EPS) basically provides packet-optimized system; higher-data-rate; lower-latency [8]. The importance of EPS studies is on improving Packet Switched technology for catering to the needs of fast growing IP traffic [9]. Hence, the prime goals of EPS are to:

- Furnish extensive rate of data transmission with reduced latency, enhanced Quality of Service (QoS) and maximum level of security.

- Should have support for multiple access systems (both legacy and new) for assuring better mobility and service spontaneity among the access systems. 
- $\quad$ Should have support for various user preferences, policies coined by operator, and conditions of access network.

- Invoke enhancement of the fundamental system performance with extended capability of migration to EPS.

\subsection{Long-Term Evolution}

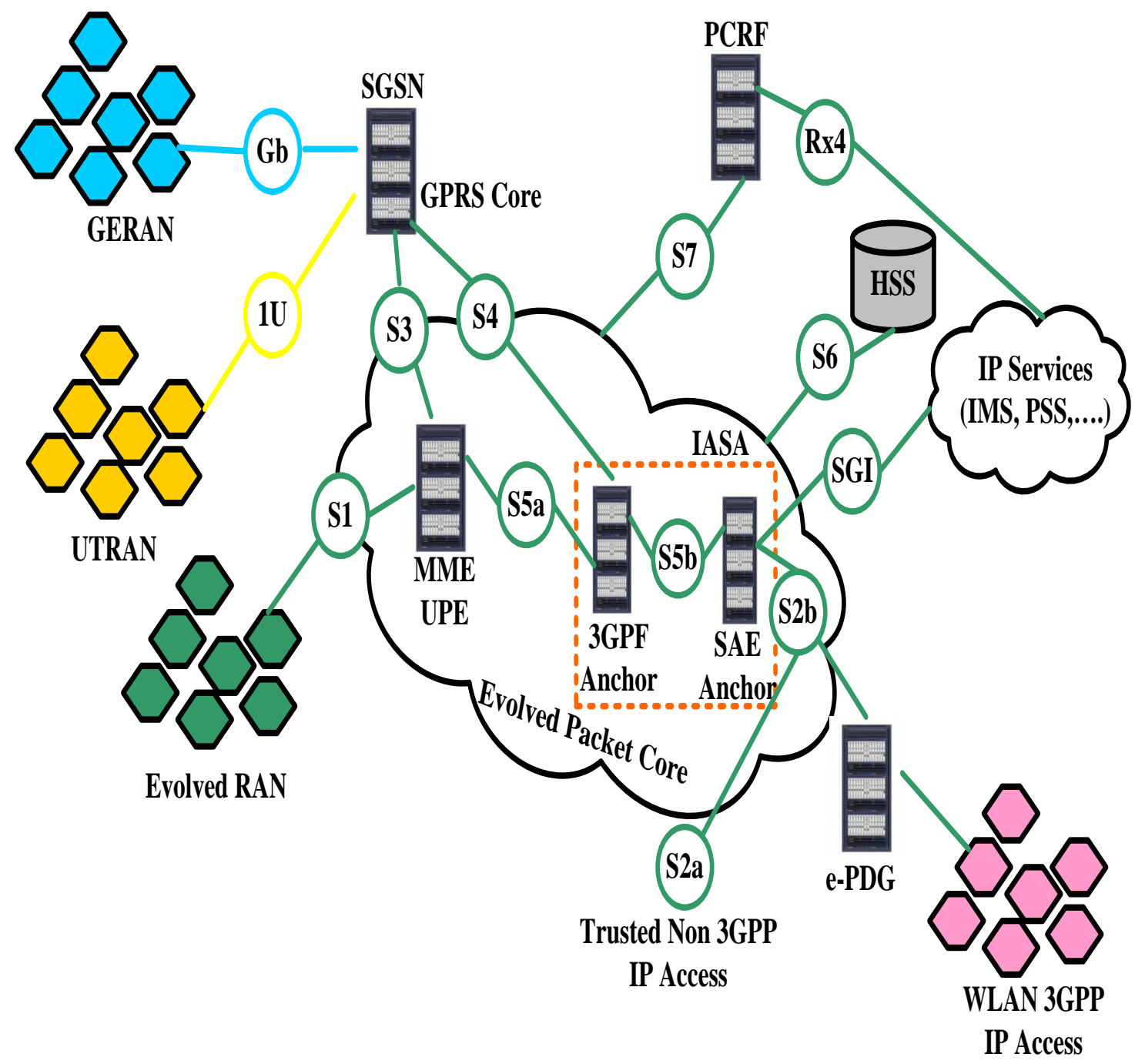

The study towards Long-Term Evolution (LTE) started in 2004 with the objective of selecting technologies for retaining 3GPP's Universal Mobile Telecommunications System (UMTS) at the forefront of mobile wireless well into the next decade. Prime motive behind these were i) spectral efficiency, ii) overall system cost, iii) peak data throughput, iv) device complexity, v) latency, and vi) flexible channel bandwidth.

Fig 1: Schema of LTE [10]

\subsection{Quality of Service}

Quality of Service parameters play a critical role in the success of the EPS specified for GSM and UMTS. The technical meanings of these Quality of Service (QoS) features are:

\subsubsection{Network Access}

By notice the network indication symbol on the mobile display signals that he can use the service of this network operator (or any other means to indicate to the user that a network is available).

\subsubsection{Service Access}

Service provider has to provide the service to its user without any delay in time when service is needed.

\subsubsection{Service Integrity}

It's the Quality of Service during service usage.

\subsubsection{Service Retain ability}

Service Retain ability defines the termination of services (based on the favorable or against the determination of the client).

\subsection{Heterogeneous Characteristics:}

The EPS support mobility between heterogeneous access systems (PSTN-Public switched telephone network), Internet, 3GPP, UTRAN, etc). In the EPS system to avoid the service degradation mobility mechanism is supported in the event of handover within and across 3GPP legacy systems or E-UTRAN and non-3GPP access systems. The Evolved Packet System (EPS) also supports a mobility mechanism that accommodates multiple versions of access systems. 


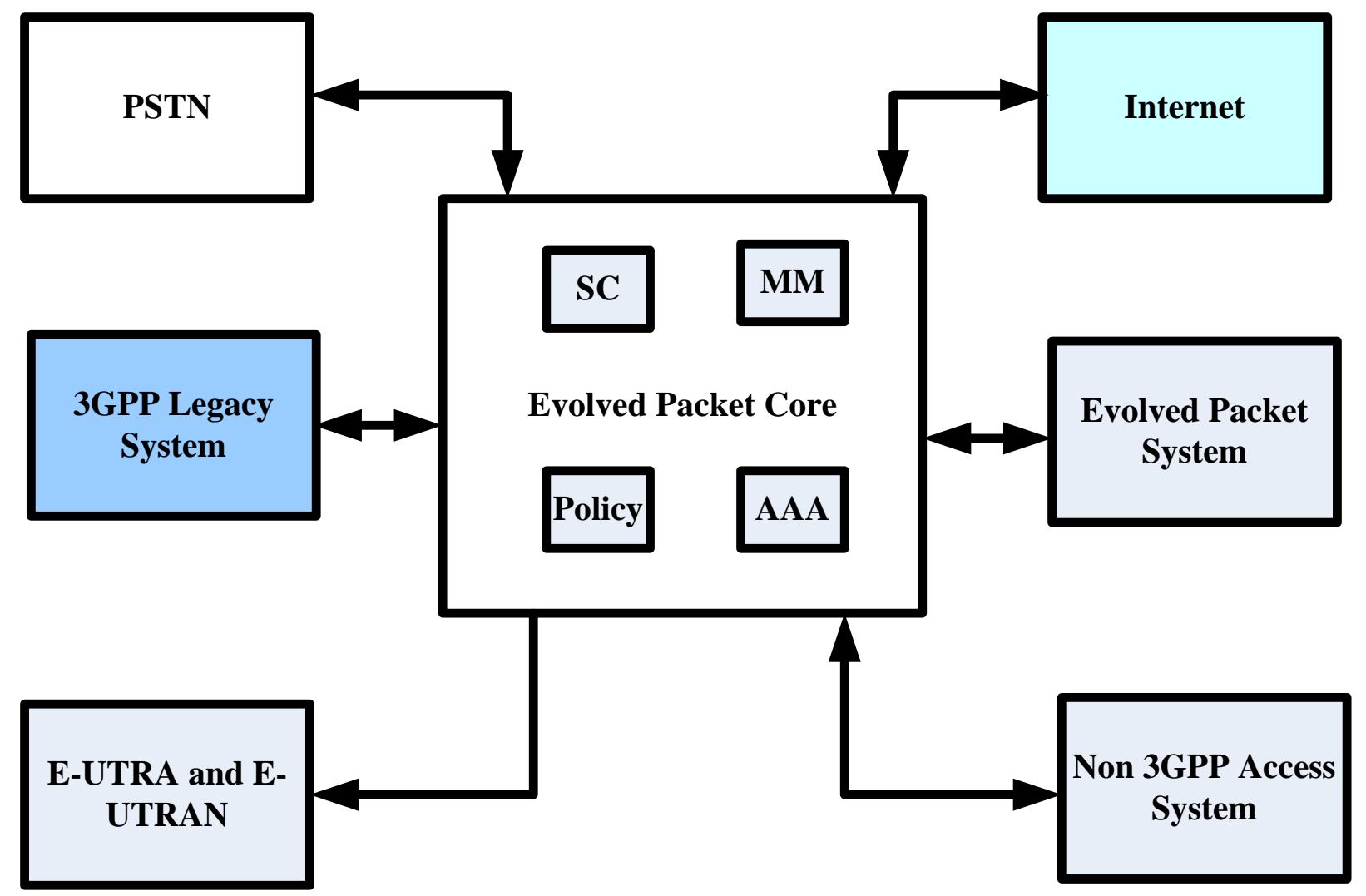

Fig 2: Heterogeneous access system mobility

\subsection{Performance Requirements for EPS}

Evolved packet System need following performance objective like higher user data rates, improved system capacity and coverage, lower operating costs, reduced network complexity and, reduced latency. The Evolved Packet System (EPS) has to meet or exceed the below discussed performance criteria:

- $\quad$ The radio access network should be capable of supporting spontaneous peak packet data rates of $100 \mathrm{Mbps}$ on the radio access bearer downlink and 50 Mbps on the uplink.

- Compared to existing 3GPP access networks, Evolved Packet System (EPS) should be capable of reducing users and control plane latency.
- The system should be capable of supporting massive load and avoid congestion too.

- The level of mobility management signaling and system complexity should reduce operating costs and infrastructure.

The area of LTE in research community is not a new aspect, but various researchers have attempted to enhance the EPS system. Table 1 summarizes all the reviews of existing systems in the past to understand the significance of existing survey papers archived in the literature. This part of discussion in Table 1 is important to visualize the potential content enrichment and information nature of our present review paper.

Table 1. Existing Survey Papers

\begin{tabular}{|c|c|c|c|}
\hline Authors & Publications & Focus & Inference \\
\hline Chadchan \& Akki [11] & IJCEE-2010 & $\begin{array}{l}\text { Technical Overview of LTE, SAE, } \\
\text { EPS }\end{array}$ & $\begin{array}{l}\text { Pros: Good Theoretical Study } \\
\text { Cons: No discussion of Research gap, No discussion of } \\
\text { upcoming trends issues }\end{array}$ \\
\hline Akyildiz et al. [12] & Elsevier-2010 & Discussed on advanced LTE & $\begin{array}{l}\text { Pros: Elaborated and systematic review } \\
\text { Cons: Narrowed discussion on open issues and comparative } \\
\text { study }\end{array}$ \\
\hline Senger et al. [14] & IJCSE-2011 & $\begin{array}{l}\text { LTE Architecture \& different } \\
\text { multiplexing techniques on }\end{array}$ & $\begin{array}{l}\text { Pros: Brief technical discussion on LTE architectures } \\
\text { Cons: No discussion of significant related work, No discussion }\end{array}$ \\
\hline
\end{tabular}




\begin{tabular}{|c|c|c|c|}
\hline & & $\begin{array}{ll}\text { WiMAX } & \text { (Worldwide } \\
\text { Interoperability for } & \text { Microwave } \\
\text { Access) } & \end{array}$ & $\begin{array}{l}\text { on Research gap, narrowed informative contents on upcoming } \\
\text { issues. }\end{array}$ \\
\hline Schwarz et al. [15] & IEEE-2013 & LTE techniques and standards & $\begin{array}{l}\text { Pros: Discussed benefits of Vienna LTE link-level simulator } \\
\text { Cons: No discussion of significant related work, No discussion } \\
\text { on Research gap }\end{array}$ \\
\hline Yu et al. [16] & IEEE-2013 & Trunking communication over LTE & $\begin{array}{l}\text { Pros: Effective study of QoS as well as Trunking } \\
\text { Communication } \\
\text { Cons: Narrowed discussion on open issues and comparative } \\
\text { study }\end{array}$ \\
\hline Singh \& Singh [17] & IJETT-2013 & Scheduling of radio resources & $\begin{array}{l}\text { Pros: Structured discussion on downlink resource scheduling } \\
\text { algorithms } \\
\text { Cons: No investigation towards research gap }\end{array}$ \\
\hline Madi et al. [19] & JAS-2014 & Multi-cell packet scheduling & $\begin{array}{l}\text { Pros: Good study of network QoS, scheduling algorithms. } \\
\text { Cons: Narrowed discussion on open issues }\end{array}$ \\
\hline Wang et al. [20] & IJSC-2014 & Architecture security & $\begin{array}{l}\text { Pros: Discussed LTE security architecture with its drawbacks, } \\
\text { Discussed open issues } \\
\text { Cons: Narrowed discussion of open issues }\end{array}$ \\
\hline Boujelben et al. [21] & IOSR-2014 & $\begin{array}{l}\text { Existing interference mitigation } \\
\text { techniques for LTE }\end{array}$ & $\begin{array}{l}\text { Pros: Performed comparative study on schemes } \\
\text { Cons: No discussion of open issues }\end{array}$ \\
\hline Lee et al. [22] & IEEE-2014 & Radio resource management & $\begin{array}{l}\text { Pros: Good discussion on ongoing research on resource } \\
\text { management, systematic and updated study on scheduling } \\
\text { algorithms, resource allocations. }\end{array}$ \\
\hline
\end{tabular}

\section{RECENT STUDY}

This section discusses about the recent research techniques that were published from 2010 till date. Zulhasnine et al. [24] have discussed the spectrum sharing problems on 3GPP-LTE cellular network and analyzed the interference issues owing to faulty transmitter design. Lai et al. [25] have presented an architecture for performing efficient hand-off between IEEE 802.11 and EPS networks using optimized proxy mobile IPv6. The work done by John and Ventura [26] are also seen to use proxy mobile IPv6 to normalize WLAN (Wireless Local Area Network) traffic and attain IP flow mobility. Study toward incorporating access policy and security was seen in the paper of Li et al. [27]. The authors have deployed standard cryptographic principles to enhance the authentication process of $3 \mathrm{G}$ networks. The issue of mobile service is emphasized by the research attempt of Ahmed and Choukair [28] for EPS based services. Although the authors discussed that their technique can considerably reduce congestion, there was no significant outcome to prove it. Wang et al. [29] have quantitatively analyzed the transmission behavior of the LTE network using statistical analysis. The authors have considered path-loss and coherence bandwidth to evaluate the outcome accomplished from their study. Aiash et al.
[30] have proposed a framework for supporting heterogeneous characteristics of LTE networks. The study showed that YComm framework is highly suitable to analyze LTE network elements. Significant studies of LTE network from the viewpoint of cloud computing was done by Guasch [31]. The author has studied the virtualization aspects of the LTE network. Swetha et al. [32] have studied the scheduling problems in LTE network considering video traffic and eventually proposed a fair scheduling technique using round robin to enhance the quality of experience (QoE). Study in similar direction considering scheduling issues was also addressed by Sharma and Chopra [33]. The packet scheduling issues were studied using various multimedia files like voice, video, text transmission over LTE networks. Table 1 summarizes the significant contributions of the recent studies along with brief highlights of their techniques and inference for better visibility of the effectiveness of their contribution. Section V discusses the cumulative research gap considering this part of the study too. 
Table 2. Significant Contribution of Recent Studies

\begin{tabular}{|c|c|c|c|}
\hline Authors & Problems & Techniques & Inference \\
\hline $\begin{array}{l}\text { Zulhasnine et al. } \\
\text { [24] }\end{array}$ & $\begin{array}{l}\text { Resource Allocation in LTE } \\
\text { network }\end{array}$ & $\begin{array}{l}\text { Greedy Heuristics approach for resource } \\
\text { block selection }\end{array}$ & Outcome of the result is not benchmarked \\
\hline Lai et al. [25] & $\begin{array}{l}\text { Efficient handoff in EPS } \\
\text { network }\end{array}$ & $\begin{array}{l}\text { Optimization of PMIPv6 (Proxy Mobile } \\
\text { IPv6) }\end{array}$ & $\begin{array}{l}\text { Less handoff delay, reduced packet loss \& } \\
\text { signaling overhead. } \\
\text { Study not evaluated with uplink/downlink } \\
\text { transmission }\end{array}$ \\
\hline $\begin{array}{l}\text { John \& } \quad \text { Ventura } \\
{[26]}\end{array}$ & IP flow mobility & $\begin{array}{l}\text { Network based solution on PMIPv6 } \\
\text { interface }\end{array}$ & $\begin{array}{l}\text { Outcomes neither discussed effectively nor } \\
\text { benchmarked }\end{array}$ \\
\hline Lu et al. [27] & $\begin{array}{l}\text { Effective Access policy in } \\
\text { mobile communication }\end{array}$ & $\begin{array}{l}\text { Standard cryptographic technique using } \\
\text { SHA }\end{array}$ & Narrowed scope of outcomes \\
\hline $\begin{array}{ll}\text { Ahmed } & \& \\
\text { Choukair [28] } & \end{array}$ & $\begin{array}{l}\text { Improve mobile service } \\
\text { dependability in LTE network }\end{array}$ & $\begin{array}{l}\text { Policy and charging control for better } \\
\text { QoS }\end{array}$ & Narrowed scope of outcomes \\
\hline Wang et al. [29] & $\begin{array}{l}\text { Evaluating indoor channels in } \\
\text { LTE network }\end{array}$ & Distance-dependent path-loss model & $\begin{array}{l}\text { Statistical results showed } \\
\text { better coherence } \\
\text { bandwidth. However, } \\
\text { benchmarked }\end{array}$ \\
\hline Aiash et al. [30] & $\begin{array}{l}\text { LTE network supportability in } \\
\text { Oheterogeneous environment }\end{array}$ & Discussed relationship between LTE and & -Comm infrastructure \\
\hline Guasch [31] & Virtualization of LTE network & Building EPC test bed in data center & Study focuses mainly on virtualization schemes \\
\hline Swetha et al. [32] & $\begin{array}{l}\text { Scheduling issues in LTE } \\
\text { network }\end{array}$ & $\begin{array}{l}\text { Comparative evaluation using EXata } \\
\text { 4.1network simulator }\end{array}$ & $\begin{array}{l}\text { Round Robin is better for scheduling. Result } \\
\text { has less scope of optimization in heavy traffic. }\end{array}$ \\
\hline $\begin{array}{l}\text { Sharma \& Chopra } \\
\text { [33] }\end{array}$ & $\begin{array}{l}\text { Scheduling issues in LTE } \\
\text { network }\end{array}$ & Designed a simple scheduling algorithm & $\begin{array}{l}\text { Optimal throughput and delay seen. Results not } \\
\text { benchmarked. }\end{array}$ \\
\hline
\end{tabular}

\section{EMERGING TREND OF RESEARCH}

The previous section has discussed about the various significant studies, where it can be seen that there exists massive survey work towards investigation of Evolved Packet System (EPS) (Table.1) as well as some recent potential studies with brief discussion of various techniques implemented (Table.2). This section discusses about some of the evolving trends that have been witnessed by the research community. Hence, for this purpose, we choose to perform a close scrutiny of those concepts involved in existing standard publications that seemed to be slowly gaining pace among the researchers.

\subsection{Evaluation of MIMO Technique}

The design principle based on MIMO techniques calls for using multiple antennas in the side of transmitter, receiver, as well as both. The study performed by Paul [34] in 2010 discusses about the in-depth analysis as well as performance evaluation of the LTE downlink with MIMO (multiple input, multiple output) antennas. The outcome of their study proved that it is possible to meet the design requirements of 3GPP LTE with higher certainty and reliability. The analytical outcomes of the study have also suggested that spatial multiplexing is one of the ideal processes for accomplishing higher peak rates while transmitting diversity is an important policy for minimizing the bit error rate thereby enhancing quality of signal. Although the study was interesting, there is a need to perform analysis based on multiple users in the MIMO scheme as the study was focused on single user. Moreover, there is also a need to understand the switching capabilities without which the LTE downlink performance would not be effectively measured. However, the work is highly inspiring as it gave the idea of designing a system for evaluating performance of LTE downlink over various channel modes and user behavior. In the same year (2010), Ketonen et al. [35] have also studied in the similar direction for understanding the performance of LTE receivers. The study was done considering FPGA and ASIC implementation by adopting linear minimum mean-square error and k-best list sphere detector. The authors have considered designing algorithm using an OFDM based MIMO transmission system. Synthesized on Xilinx, the outcome of the study was witnessed with better throughput. Still, the evaluation techniques could be further enhanced by redefining the architecture as well as switching techniques for more sophisticated detectors using channel properties. Hence, more techniques as well as algorithms for evolving packet system is required that could have better visualization of performance parameters exclusively for MIMO based consideration in LTE network.

\subsection{MIMO-OFDM}

The constant change in the customer requirements along with technological advancement has reasoned for evolution of $2 \mathrm{G}$ (second generation), 3G (third generation), and 4G (fourth generation) networks. Along with the evolving needs, the complexity of the future generation of the wireless communication system is drastically growing globally. Hence, from the year 2010 onwards, we have come across several research papers that have tried to emphasize that by integrating MIMO system with OFDM; various issues of furnishing better data rates under frequency-selective fading channel could be addressed. One of such work is found in Gomez et al. [36]. The detailed analysis of functionality such has channel estimation, MIMO detection, channel coding, MIMO transmission modes and cross-layer scheduling is required for design and evaluation of MIMO-OFDM . Using various parameters like BER (Bit Error Rate), ASE (Asymptotic Standard Error), SINR (Signal-to- 
Interference-plus-Noise Ratio), delay, load, etc were evaluated to ensure the better outcome of the study. An extended study in similar direction in the same year 2010 was discussed by Hanzo et al. [37]. In 2011, Simko et al. [38] developed an algorithm for estimation of ICI that can be useful to OFDM systems with an arbitrary pilot structure. The estimator models the channel variation by means of a basis expansion model (BEM). The performance of the estimator and of the subsequent equalization is evaluated in an UMTS (Universal Mobile Telecommunications System) long term evolution (LTE) link level simulator. However, focus of [38] was less on the characteristics of MIMO and more on OFDM. The study performed by Omri et al. [39] in 2011 have used neural network for evaluating performance of downlink LTE network with respect to MIMO-OFDM inclusion. Open issues of [39] and [40] were not investigated for any possible relationship between various channel models in MIMO as well as OFDM with LTE networks. A study performed by Jessen [40] in 2012 in his report of Aalborg University have addressed in this point. The researchers have considered using Turbo coding and rate matching complying to LTE standards and fading channels to understand the influence of channel models in MIMO-OFDM exclusively for LTE networks. Another study in similar direction was discussed by Oborina [41] in her thesis of Aalto University in 2012. The authors have studied multi-stream multi-carrier wireless transmission considering realistic mobility scenario. The importance of contributing factor of MIMO-OFDM in 4G LTE was also emphasized by Patil [42] in 2013. The recent work of Kildal et al. [43] in 2014 have explicitly discussed about the introduction of a systematic approach to include the statistical properties of the user and his or her terminal, when characterizing the performance of $5 \mathrm{G}$ networks.

\subsection{Evaluation of Spectral Efficiency}

It is understood that the spectral efficiency of the cooperative multi-hop communication as well as network coverage should be optimally high. The evaluation of the spectral efficiency was carried out by Haider et al. [44] in 2011. The authors have investigated the spectral efficiency for Mobile Femtocell deployment cellular systems with two resource partitioning schemes. The evaluation test-bed consists of blocks of 12 contiguous sub-carriers in the frequency domain and 7 OFDM symbols in the time domain. One sub-frame $(1 \mathrm{~ms})$ is regarded as scheduling period. The carrier bandwidth is fixed at $10 \mathrm{MHz}$ with 50 RBs. The study has also carried out analysis of spectral efficiency of the orthogonal as well as non-orthogonal schemes. The study outcomes are quite optimistic and interestingly supportive enough for LTE networks. However, after this, no significant study was found to discuss about spectral efficiency of LTE networks. Hence, this is one of the emerging trends of the research community that should require further investigation. An efficient discussion of spectral analysis will elicit more latent behavior of LTE networks from the study viewpoint. Although study considering QoS parameters (throughput and BER, SNR BER, ASE, SINR, delay) in LTE network have also been done in past, but very little work is found (e.g. Ahmed \& Choukair [28].

\subsection{Massive Transmission and Traffic Behavior}

It is said that multimedia files are the heavier files that are transmitted on the networks. Hence, various system performances ensuring the quality of the wireless network and its supportability towards quality of service parameters are highly essential. Research in this direction was carried out by Shuaib and Sallabi [45] in 2010. To manage the transmission rates in MPEG-4 and H.264 video over a QoS-based wireless LTE network authors designed a semi-optimal video smoothing approach. In this technique smoothing buffer with pre-defined thresholds is used to smooth the transmission rates by that minimum information of the video is transmiting. The authors have used channel feedback based analysis. However, extended evaluation is required to understand the traffic behavior as well as massive transmission while streaming in LTE networks, which is still an open issue. Shin et al. [46] in 2013 have emphasized on 4G systems where evaluation of data and signaling overhead, handover latency, and packet delay play a crucial role. Han et al. [47] in 2013 have classified existing content delivery acceleration solutions in mobile networks into three categories: mobile system evolution, content and network optimization, and mobile data offloading, and provide an overview of available solutions in each category. Now it is known that mobile video applications generate the largest wireless data traffic volume. Different video applications behave differently in term of bandwidth consumption. Although, some authors have started investigating about it, but the extent of technological enriched standards and benchmarked techniques are yet missing.

\section{RESEARCH GAP}

The discussion points highlighted in Table 1, Table 2, and previous sections highlight that there are extensive archival of literature in the past that have attempted to investigate for enhancing the issues associated with upcoming LTE networks. The research gaps explored from the entire review of literature are as follows:

\subsection{Few Standardization}

We found that although various attempts of research work exist till last decade, but extent of research work is more from 2010 onwards till 2014. Moreover, it is found that researchers have analyzed and claimed the effectiveness of their outcomes in their considered scenario without much comparing their outcomes with some other researchers' work. Hence, the absence of such comparative performance results in lack of standardization resulting in less scope of performing benchmarking of the future research outcomes. The applicability of the existing studies towards LTE network is significantly less owing to less standard framework and benchmarked studies posing as potential research gap.

\subsection{Insufficient Study of Mobile Networks}

At present with the dynamic increase of the customer base on mobile networks, even $3 \mathrm{G}$ cannot cater to the requirements of the customers. Hence, since the last 5 years, both the customers and the service providers felt the emergence of $4 \mathrm{G}$ networks from $3 \mathrm{G}$ network for the purpose of meeting the increasing demands of traffic and satisfactory user-experience. It was seen that various studies have considered the scenario of mobility in their simulation based studies that have attempted to discuss the delay factor in the mobile networks and paved a path for future research direction, thereby enhancing the path of 4G LTE networks. Observing the shortcomings of existing mobile networks, various solutions have been proposed to reduce content delivery latency and enhance subscribers' QoS in mobile networks. It was found that majority of such research attempts have adopted the centralized mobility management schemes and less of distributed management schemes (Internet). Adoption of such schemes can never cater to the needs of massive transmission and ensure optimal traffic behavior. It is applicable in the case of video transmission in LTE. Moreover, the studies pertaining to distributed management schemes (Shin et al. [46]) has considered dynamic consideration of the traffic, which is missing. None of the studies are found to model standards or protocols for mapping uncertain traffic behavior of the networks. The existing studies are found partially successful in optimally reducing the traffic volume over mobile networks. For this reason, the associated 
issues of accelerating content delivery, mitigating traffic congestion evolution were never addressed in any significant studies till date. The discussion as well as analysis of mobile networks for LTE is merely discussed with iterative finite number of mobile users under various circumstances of uniform velocity, which makes the studies much narrow. All the studies seem to understand the user mobility behavior, whereas in real time, the mobility behavior is something that is never possible to be determined. One good example can be cited for this issue in Mobile Adhoc Network, where the nodes are in consistent movement in random direction. Hence, without considering such dynamic topology considering real-time constraint and performing the analysis without benchmarking raise the question of outcome reliability.

\subsection{Narrow Scope}

It was seen that majority of the study has focused on evaluating LTE networks considering downlink transmission. It should be known that a network behavior and performance can only be evaluated when uplink transmission is also considered at the same time. Hence, there is a serious need to have such consideration while working on a model for LTE networks. The problem becomes much worse when heterogeneity of the network protocol for EPS and UTRAN is considered. Hence, there is a potential need of novel LTE architecture for performing an extensive evaluation considering various broader cases of transmission for large network.

\subsection{Evaluating Fault Tolerant Transmission}

As stated in a prior section that a network could be closely analyzed for its sustenance in successful transmission with better QoS, if the file system considered for transmission is multimedia. As seen in the literature there are very few significant research attempts where authors have considered video transmission. Thus, a significant research gap is observed where less extent of investigation is done for LTE networks considering multimedia transmission. Almost no studies were found that have considered real-time streaming of the multimedia files. We feel that this point is most important to be highlighted as majority of the customers that want to use $4 \mathrm{G}$ networks have higher probability to use video calls (live streaming) or use certain peer-to-peer applications (Skype) or remote system control (Team Viewer) from their Smart phones consuming enough bandwidth as compared to usage of other application. Hence, such issues should significantly address to mitigate the problems.

There are various loopholes in the existing studies that can be summarized as: i) few benchmarking works, ii) simulation parameters selected have narrow scope in outcome visualization, iii) studies focused on selective environment of the authors that are usually not applicable in real-life, iv) less extent of standardization, v) few studies toward fault tolerant features, vi) insufficient studies toward mobile networks using MIMO and OFDM principles, vii) less investigation done in effective spectral analysis for dynamic traffic scenario, viii) absence of mathematical modeling to script the real-time and uncertain traffic behavior over LTE networks, ix) less compatibility of existing solutions with actual device requirements (transcoding), and $\mathrm{x}$ ) inadequate research towards MIMO principle as well as single carrier FDMA to upgrade the network performance.

\section{CONCLUSION}

This paper has discussed various significant contribution of the past research attempts. The prime aim of the paper was to understand the effectiveness in the techniques for improving the LTE network from the viewpoint of evolving packet system. It was also found that there were significant studies done towards reviewing the existing studies too. We have discussed even the review papers in our work in Table 1 to highlight that the prior review papers have theoretical discussion of existing techniques where the investigation towards standard and benchmarking work were never found to address. This paper performs in-depth understanding of the existing work in the process of exploring the open issues, emerging trends and significant research gap and hence, this review paper furnishes better comparative analysis of the techniques and highlights their effectiveness in improving the LTE network for the future wireless communication systems. The points mentioned in this paper will be highly helpful for the future researchers to understand the significant of EPS, LTE, and UTRAN and visualize the original need of the design formulation for further enhancing its performance. Our work towards future direction will be to design an efficient framework that can address the research gap highlighted in this paper. We are currently investigating the possible design of e-UTRAN system whereby our design principles consider OFDM for downlink transmission, single carrier FDMA for uplink transmission and employ MIMO to further enhance the throughput under various dynamic traffic conditions.

\section{REFERENCES}

[1] XU, X.2004.Interdisciplinary Mobile Media and Communications: Social, Political, and Economic Implications. Technology \& Engineering. pp. 409

[2] Sesia, S., Toufik, I., Bake, M.(2011). LTE - The UMTS Long Term Evolution: From Theory to Practice. Wiley Publication

[3] Saily, M., SÉbire, G., Riddington, and E.2010.GSM/EDGE: Evolution and Performance. Wiley Publication

[4] Yi, S., Chun, S., Lee, Y.D., Park, S.J., Jung, S.H.2012. Radio Protocols for LTE and LTE-Advanced.Wiley publication

[5] Myung, H.G., Goodman, D.J.2008. Single Carrier FDMA: A New Air Interface for Long Term Evolution. Wiley Publication

[6] Wisely, D., Eardley, P., Burness, L.2002. IP for 3G: Networking Technologies for Mobile Communications. John Wiley \& Sons. pp. 295

[7] Payaswini, P., Manjaiah, D.H.2013. Challenges and issues in $4 \mathrm{G}$ - Networks Mobility Management. International Journal of Computer Trends and Technology (IJCTT). Vol.4 Iss. 5

[8] Lescuyer, P., Lucidarme, T. 2008. Evolved Packet System (EPS): The LTE and SAE Evolution of 3G UMTS. Wiley \& Sons. Copyright

[9] Eumney, M.2008. LTE and the Evolution to 4G Wireless: Design and Measurement Challenges. Wiley \& Sons. Copyright

[10] ]http://www.commverge.com/Solutions/BroadbandAccessN etworks/LongTermEvolutionLTE/tabid/161/Default.aspx

[11] Chadchan, S. M., and Akki, C. B.2010. 3GPP LTE/SAE: An Overview. International Journal of Computer and Electrical Engineering, Vol. 2, No. 5, pp. 1793-8163

[12] Akyildiz, I. F., G-Estevez, D.M., Reyes, E.C.2010. The evolution to $4 \mathrm{G}$ cellular systems: LTE-Advanced. Physical Communication, pp. 3217-244

[13] Ciochina, C., and Sari, H.2010. A Review of OFDMA and Single-Carrier FDMA and Some Recent Results. Advances in Electronics and Telecommunications. Vol. 1, No. 1 
[14] Sengar, S.S., Singh, A., Tripathi, P.N.2011. A Survey on Telecommunication Technology Standards. International Journal on Computer Science and Engineering (IJCSE), Vol. 3 No. 5

[15] Schwarz, S., Ikuno, J.C., Simko, M. , Taranetz, M., Wang, Q., and Rupp, M.2013. Pushing the Limits of LTE: A Survey on Research Enhancing the Standard. Accepted for publication in IEEE Access

[16] Yuy, H., Sunz, S., Yu, Q., He, C., Meng, W., Tan, X., Zhang, Z.2013. Survey of Trunking Communications over LTE: Implementation Framework, Application Requirements, and Quality of Service.IEEE Wireless Communications and Networking Conference (WCNC): Services \& Applications

[17] Singh, D., Singh, P.2013. Radio Resource Scheduling in 3GPP LTE: A Review. International Journal of Engineering Trends and Technology (IJETT), Vol. 4 Iss. 6

[18] Adu, O.I., Oshin, B.O., and Alatishe, A.A.2013. VoIP on 3GPP LTE Network: A Survey. Journal of Information Engineering and Applications, Vol.3, No.11

[19] Madi, N.K.M., Hanapi, Z.B.M., Othman, M., and Subramaniam, S.2014. On Multi-cell Packet Scheduling of LTE-A Cellular Networks: A Survey of Concepts Related Challenges and Solutions. Journal of Applied Sciences. Vol. 14 , Issue. 20 , pp. $2422-2438$

[20] Wang, J., Zhang, Z., Ren, Y., Li, B., and Kim, J-U.2014. Issues toward Networks Architecture Security for LTE and LTE-A Networks. International Journal of Security and Its Applications. Vol.8, No.4, pp.17-24

[21] Boujelben, M., Benrejeb, S., Tabbane, S.2014. Interference Coordination Schemes for Wireless Mobile Advanced Systems: A Survey. Journal of Electronics and Communication Engineering (IOSR-JECE), Vol. 9, Iss. 1, pp. $80-90$

[22] Lee, H., Vahid, S., and Moessner, K.2013. A Survey of Radio Resource Management for Spectrum Aggregation in LTE-Advanced. Institute of Electral and Electronic Engineers, pp. 1-16

[23] Lee, Y.L., Chuah, T. C., Loo, J., and Vinel, A.2014. Recent Advances in Radio Resource Management for Heterogeneous LTE / LTE-A Networks. Institute of Electral and Electronic Engineers

[24] Zulhasnine, M., Huang, C., and Srinivasan, A.2010 Efficient resource allocation for device-to-device communication underlaying LTE network. In Wireless and Mobile Computing, Networking and Communications (WiMob), IEEE 6th International Conference. pp. 368-375

[25] Lai, C., Li, H., Zhang, Y., Cao, J.2011. A Fast Seamless Handoff Scheme between IEEE 802.11 and EPS Networks Using Optimized Proxy Mobile IPv6. International Journal of Digital Content Technology and its Applications. Vol. 5, No. 8

[26] John, C.T., and Ventura, N.2011. WLAN Offload and IP Flow Mobility in the Evolved Packet Core. SATNAC 2011, East London International Convention Centre, South Africa

[27] Xinghua, L., Jianfeng, M., YoungHo, P., and Li, X.2010. A USIM-based uniform access authentication framework in mobile communication. EURASIP Journal on Wireless Communications and Networking
[28] Ahmed, O. B., and Choukair, Z.2013. QOS-Apcvs: An Enhanced EPS-IMS PCC Architecture Proposal to Improve Mobile Service Dependability. Electrical \& Computer Engineering: An International Journal (ECIJ). Vol. 2, No. 4

[29] Wang, Y., Lu, W-J., and Zhu, H-B.2013. Propagation Characteristics of the LTE Indoor Radio Channel With Persons at $2.6 \mathrm{GHz}$. IEEE Antennas and Wireless Propagation Letters. Vol. 12

[30] Mahdi, A., Mapp, G., Lasebae, A., and Nemrat, A.2013.Supporting LTE Networks in Heterogeneous Environment Using the Y-Comm Framework. InComputer Networks \& Communications (NetCom). pp. 125-136

[31] Guasch, V. F.2013. LTE network Virtualization. School of Electrical Engineering

[32] Swetha, N., Mohankumar, M., Devaraju, J.T.2014. Performance Study of Round Robin and Proportional Fair Scheduling Algorithms by Emulation for Video Traffic in LTE Networks. International Journal of Computer Application

[33] Sharma, V., Chopra, P.K2014. Performance Analysis of A Novel Scheduling Algorithm for Different Traffics in LTE Networks. International Journal of Computer Science and Information Technologies. Vol. 5 (5), pp. 6631-6634

[34] Kebede, G.M., Paul, O. O.2010. Performance Evaluation of LTE Downlink with MIMO Techniques. Master Thesis Electrical Engineering Thesis no: MEE10:104

[35] ]Ketonen, J., Juntti, M., and Cavallaro, J.R.2010 Performance-Complexity Comparison of Receivers for a LTE MIMO-OFDM System. IEEE Transactions on Signal Processing, Vol. 58, No. 6

[36] Gomez,G.,im’enez,D.M.,.S-Sanchez,J.J.,and J.T.Entrambasaguas.2010. ANext GenerationWireless Simulator Based on MIMO-OFDM: LTE Case Study. EURASIP Journal onWireless Communications and Networking.Article ID 161642, pp. 14

[37] Hanzo, L., Akhtman, Y., Jiang, M., and Wang, L.2010. MIMO-OFDM for LTE, WIFI and WIMAX: Coherent versus Non-Coherent and Cooperative Turbo-Transceivers. John Wiley/ \& Sons

[38] Simko, M., Mehlf uhrer, C., Zemen, T., and Rupp, M.2011. Inter-Carrier Interference Estimation in MIMO OFDM Systems with Arbitrary Pilot Structure. In Vehicular Technology Conference (VTC Spring), IEEE 73rd, pp. 1-5

[39] Omri, A., Hamila, R., Hasna, M., Bouallegue, R., and Chamkhia, H.2011. Estimation of highly selective channels for downlink LTE MIMO-OFDM system by a robust neural network. Journal of Ubiquitous Systems and Pervasive Networks. Vol. 2, No. 1, pp. 31-38

[40] Jessen, T.2012. Impact of channel models in MIMO OFDM for LTE. Aalborg University, Doctorates Thesis

[41] Alexandra, O.2012. Analyzing performance of mobile MIMO-OFDM wireless systems: Tools and results. Department of Signal Processing and Acoustics, Doctorial Dissertations

[42] Patil, P.K.2013. Role of Contributing Factors MIMOOFDM in 4G-LTE Wireless Transmission Technologies from Technical Perspective. International Journal of Advanced Research in Computer and Communication Engineering. Vol. 2, Iss. 7 
[43] Kildal, P.S., Chen, X., Gustafsson, M., Shen, Z.2014. MIMO Characterization on System Level of 5G Microbase Stations Subject to Randomness in LOS. Access, IEEE, Vol.2, pp.1064-1077

[44] Haider, F., Wang, H., Haas, H., Yuan, D., Gao, X., You, XH., and Hepsaydir, E. 2011. Spectral efficiency analysis of mobile femtocell based cellular systems. In Communication Technology (ICCT), IEEE 13th International Conference. pp. 347-351
[45] Shuaib, K.., and Sallabi, F.2010. Smoothing of Video Transmission Rates for an LTE Network. IEEE International Workshop on Selected Topics in Mobile and Wireless Computing

[46] Shin, D-H., Moses, D., Venkatachalam, M., and Bagchi, S.2013. Distributed mobility management for efficient video delivery over all-IP mobile networks: Competing approaches. Network, IEEE, Vol. 27, No. 2, pp. 28-33

[47] Han, T., Ansari, N., Wu, M., and Yu, H.2013. On Accelerating Content Delivery in Mobile Networks. IEEE Communications Surveys \& Tutorials, Vol. 15, No. 3 